\title{
Does hydroxyapatite coating have no advantage over porous coating in primary total hip arthroplasty? A meta-analysis
}

\author{
Yun-Lin Chen, Tiao Lin, An Liu, Ming-Min Shi, Bin Hu, Zhong-li Shi and Shi-Gui Yan*
}

\begin{abstract}
There are some arguments between the use of hydroxyapatite and porous coating. Some studies have shown that there is no difference between these two coatings in total hip arthroplasty (THA), while several other studies have shown that hydroxyapatite has advantages over the porous one. We have collected the studies in Pubmed, MEDLINE, EMBASE, and the Cochrane library from the earliest possible years to present, with the search strategy of "(HA OR hydroxyapatite) AND ((total hip arthroplasty) OR (total hip replacement)) AND (RCT* OR randomiz* OR control* OR compar* OR trial*)". The randomized controlled trials and comparative observation trials that evaluated the clinical and radiographic effects between hydroxyapatite coating and porous coating were included. Our main outcome measurements were Harris hip score (HHS) and survival, while the secondary outcome measurements were osteolysis, radiolucent lines, and polyethylene wear. Twelve RCTs and 9 comparative observation trials were included. Hydroxyapatite coating could improve the HHS $(p<0.01)$, reduce the incidence of thigh pain $(p=0.01)$, and reduce the incidence of femoral osteolysis $(p=0.01)$, but hydroxyapatite coating had no advantages on survival $(p=0.32)$, polyethylene wear $(p=0.08)$, and radiolucent lines $(p=0.78)$. Hydroxyapatite coating has shown to have an advantage over porous coating. The HHS and survival was duration-dependent-if given the sufficient duration of follow-up, hydroxyapatite coating would be better than porous coating for the survival. The properties of hydroxyapatite and the implant design had influence on thigh pain incidence, femoral osteolysis, and polyethylene wear. Thickness of 50 to $80 \mu \mathrm{m}$ and purity larger than 90\% increased the thigh pain incidence. Anatomic design had less polyethylene wear.
\end{abstract}

Keywords: Hydroxyapatite, Porous, Harris hip score, Survival, Total hip arthroplasty

\section{Introduction}

Total hip arthroplasty (THA) is one of the most wide operations in orthopedic practice [1]. Cement was widely used, but high rates of failure of cemented femoral components in active patients have been reported. Cemented primary THAs showed excellent results in the shortterm but deteriorated with time, while uncemented primary THAs were not only satisfactory in the short-term but also tended to improve with time. Moreover, some studies showed that uncemented and cemented THAs had the comparable clinical results during the follow-up of 6 years, which stimulated the development of implant

\footnotetext{
* Correspondence: charleschen1991@gmail.com

Department of Orthopaedic Surgery, Second Affiliated Hospital, School of

Medicine, Zhejiang University, No.88 Jiefang Road, Hangzhou 310009, P.R. China
}

C Biomed Central

(c) 2015 Chen et al.; licensee BioMed Central. This is an Open Access article distributed under the terms of the Creative Commons Attribution License (http://creativecommons.org/licenses/by/4.0), which permits unrestricted use, distribution, and reproduction in any medium, provided the original work is properly credited. The Creative Commons Public Domain Dedication waiver (http://creativecommons.org/publicdomain/zero/1.0/) applies to the data made available in this article unless otherwise stated.

with uncemented fixation. The uncemented THAs with porous coating allowed bone ingrowth to achieve a rigid fixation. But the problem of osteolysis and stress shielding meant that the long-term stability of uncemented THA was still in question [2,3]. Meanwhile, uncemented stem fixation had the shortcoming of thigh pain. To address such problem, bioactive coating has been added to uncemented component to enhance the fixation by osseointegration of implant, of which hydroxyapatite (HA) was the most popular one.

HA coating accelerates bone healing and enhances the biologic fixation of implant due to its biocompatibility and osteoconductive potential. Several studies have shown that it could reduce the migration of HA-coated prosthetic components and have better results and higher survival rate than identical press-fit components [4]. However, 
other studies have shown that HA particles delaminated from the stem surface may induce osteolysis either by stimulating bone loss or by migration to the joint space producing third-body wear [5]. Concerns have been raised regarding the technique and parameters that were used in applying the coating to the stem as well [6]. Most reviews of clinical results, X-ray findings, and revision rates were unable to find the significant difference between hydroxyapatite and non-hydroxyapatite-coated stems [7-12]. Some studies showed that hydroxyapatite-coated components could ensure earlier return to activity, reduction in thigh pain, and fewer radiolucent lines [13-16].

A previous meta-analysis by Gandhi et al. [17] based on nine studies including 1,764 samples showed that survival from aseptic loosening had no difference between the two groups at a mean follow-up of 6.5 years, and the mean Harris hip score (HHS) between these groups demonstrated the same. Another meta-analysis by Goosen et al. [18] with eight RCTs including 857 samples reported the clinical and radiographic results, and there was no difference in HHS, endosteal bone ingrowth, and radioactive lines in the surface area of the prosthesis.

The former analysis only included four RCTs of nine studies, and one of which did not show clear HHS data, and the HA stem was grit-blasted, while porous stem was not. The latter analysis did not target new studies published in the later years. It is essential to update the previous results based on the following reasons. (1) The up-to-date 12 RCTs and comparative studies enlarged the sample size to 9,860 and expanded the population distribution. (2) Compared to the average follow-up of 5.4 years in the previous analysis, the longer 7.5 years of the new analysis may lighten on the long-term efficacy of HA coating for HHS, survival, or radiolucent lines. (3) Insufficient data in the previous meta-analysis led to the incomprehensive evaluation of the potential influential factors on HA coating effects, including the thickness and purity of HA coating, the implant design, and the duration of follow-up. The present analysis updated the meta-analysis on the effects of HA-coated stems on clinical and radiographic results, such as HHS, survival, and thigh pain incidence.

\section{Methods}

Electronic databases were searched with the limited language of English. The result was last updated on Dec. 17, 2012. The search used the following term and Boolean operators: "(HA OR hydroxyapatite) AND ((total hip arthroplasty) OR (total hip replacement)) AND (RCT* OR randomiz* OR control" OR compar" OR trial*)". The reference lists of all the selected articles were hand-searched for any additional trials. If necessary, we contacted authors to collect additional information.

The trials was included if (1) the patients had trauma, arthritis, or other diseases requiring total hip arthroplasty with age not less than 18, (2) the inclusion of the comparison between a proximally $\mathrm{HA}$ /porous coated femoral and a proximal porous coated stem who underwent primary uncemented total hip arthroplasty, (3) the measurement of outcome was at least by one of the following clinical and radiographic results, including Harris hip score, survival, thing pain incidence, radiolucent lines, femoral osteolysis, and polyethylene wear, and (4) they were published randomized controlled trials or comparison observational studies. Trials were excluded if (1) the patients underwent cemented THAs or revision uncemented THAs, (2) the trials were phase I or case report or review or animal models, or only the abstract was available, (3) the coating was only on the cup not the stem, (4) the follow-up was less than 1 year, and (5) they were sub-analysis of previously published meta-analyses.

Two of us (CYL and SMM) independently assessed each trial with a 12-item scale [19], assessing factors such as randomization, allocation concealment, and blindness. We resolved disagreements through discussion.

For each eligible trial, we extracted relevant data and checked the accuracy. In instances of unreported standard error for a mean difference in HHS, we calculated the standard error by converting the $p$ value to a $\mathrm{z}$-score and solving for the standard error with the formula: $z=$ mean difference/standard error [20]. If the article did not have a certain $p$ value and only a range, we just excluded the data $[13,21,22]$. For the trials [13], which had more than one intervention group with different ranges of HA coating, we combined group B and group C into one intervention group. For the trials [23,24], in which some identical patients were included but with different durations of follow-up, we included both of them to evaluate HHS.

Our main outcome measurements were mean postoperative HHS and the survival of prosthesis from aseptic loosening. We also assessed the incidence of thigh pain and radiographic results. To improve the clinical relevance, we used the weighed mean difference (WMD) and then estimated the relative difference in the change from baseline as the absolute benefit divided by the mean of all the baseline means of the control groups. With the fixed effect model, WMD and 95\% confidence intervals (95\% CI) were calculated and the data pooling was done using Review Manager 5.1.7.

We calculated the statistical heterogeneity using a $X^{2}$ test on $N-1$ degrees of freedom ( $N=$ sample size), with significance at 0.05 . We also assessed the inconsistency $I^{2}$ using the formula $[(Q-\mathrm{df}) / Q] \times 100 \%\left(Q=\right.$ the $\chi^{2}$ statistic, $d f=$ degree of freedom) to describe the percentage of the variability in effect estimates due to the heterogeneity [19]. We considered $I^{2}$ value of $25 \%, 50 \%$, and $75 \%$ as low, medium, and high heterogeneity, respectively. A fixed effects model was used if there was no statistical 


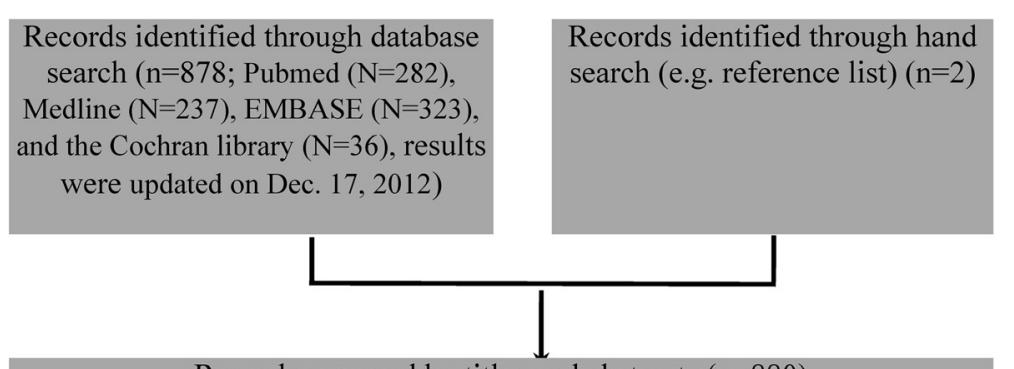

Records screened by titles and abstracts $(n=880)$

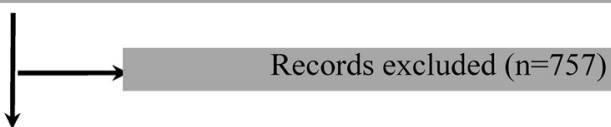

Duplicates removed $(n=70)$ and without full text article $(n=7)$

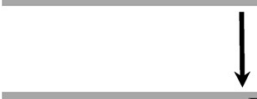

Full text assessed for eligibility $(\mathrm{n}=55)$

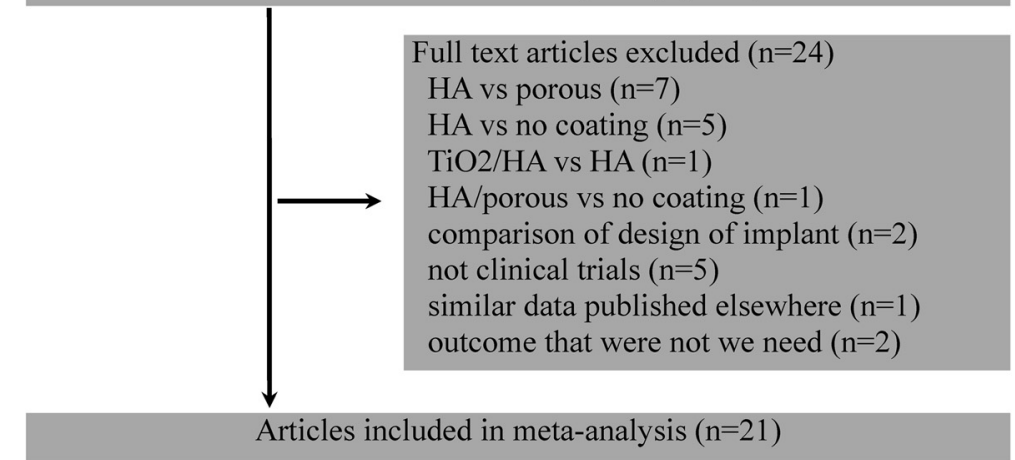

Figure $1 \mathrm{~A}$ flowchart illustrated the selection process of eligible trials in our meta-analysis.

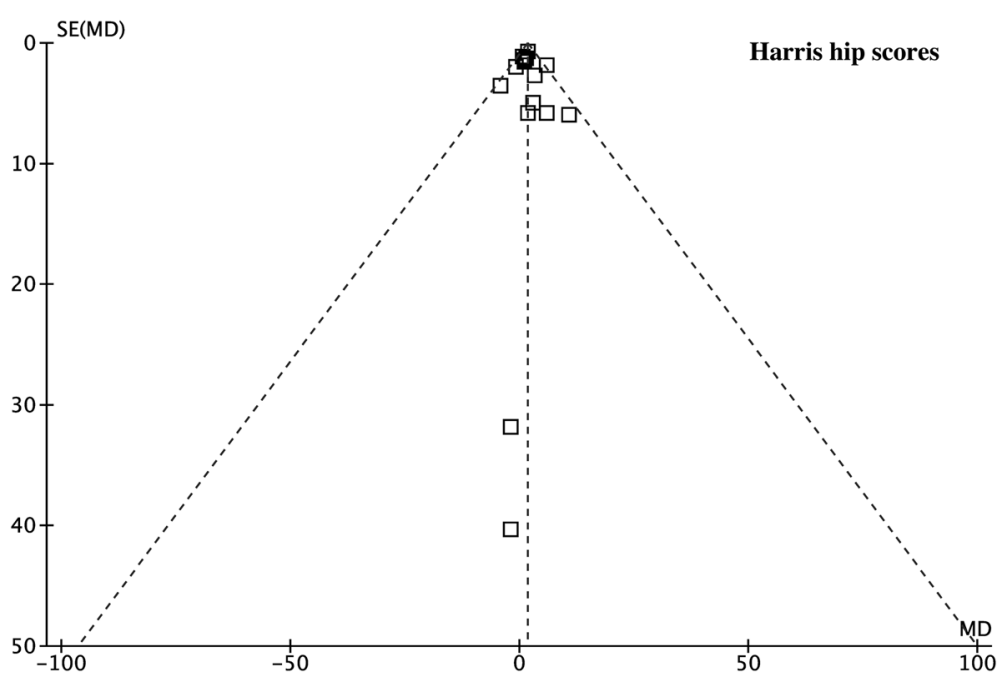

Figure 2 Funnel plot for HHS shows no publication bias. 
heterogeneity among the studies; otherwise, we used the random effects model.

We developed several subgroup analyses not only to explain the heterogeneity but also to identify the factors potentially influencing the clinical and radiographic results, such as the design of study, thickness, and purity of hydroxyapatite, follow-up duration, and implant type. Because there was revalent data showing that 50 to $80 \mu \mathrm{m}$ was the acceptable standard [25,26], 50 to $80 \mu \mathrm{m}$ was chosen to be the interval of HA thickness. Six-year

Table 1 Study characteristics

\begin{tabular}{|c|c|c|c|c|c|}
\hline Study & $\begin{array}{l}\text { Study } \\
\text { design }\end{array}$ & $\begin{array}{l}\text { Sample size } \\
\text { (HA/control) }\end{array}$ & $\begin{array}{l}\text { Mean ages of } \\
\text { patients (years) }\end{array}$ & $\begin{array}{l}\text { Sex distribution } \\
\text { (female/male) }\end{array}$ & Inclusion criteria \\
\hline Camazzola et al. [28] & $\mathrm{RCT}$ & $61(34 / 27)$ & $\begin{array}{l}48.2 \pm 9.0 / \\
50.4 \pm 8.7\end{array}$ & $22 / 39$ & $\begin{array}{l}\text { Men younger than } 60 \text { years and women younger than } \\
65 \text { years having elective primary THA }\end{array}$ \\
\hline Dorr et al. [16] & $\begin{array}{l}\text { Retrospective, } \\
\text { matched pair }\end{array}$ & $30(15 / 15)$ & $55(38-71)$ & $10 / 5$ & Patients who underwent bilateral primary THA \\
\hline $\begin{array}{l}\text { Hamadouche et al. } \\
\text { [34] }\end{array}$ & $\mathrm{RCT}$ & $45(22 / 23)$ & $65 / 64$ & $41 / 39$ & Patients with osteoarthritis of the hip requiring THA \\
\hline Incavo et al. [32] & $\mathrm{RCT}$ & $50(24 / 26)$ & 55 & $N R$ & Patients who underwent THA \\
\hline Kim et al. [23] & $\mathrm{RCT}$ & $100(50 / 50)$ & $45.3(27-61)$ & $14 / 36$ & Patients who underwent sequential bilateral primary THA \\
\hline Kim et al. [24] & $\mathrm{RCT}$ & $110(55 / 55)$ & $46.3(27-63)$ & $39 / 16$ & Patients who underwent bilateral primary THA \\
\hline $\begin{array}{l}\text { Lee and Lee } 2007 \\
\text { [33] }\end{array}$ & $\mathrm{RCT}$ & $40(20 / 20)$ & $44(25-72)$ & $2 / 18$ & $\begin{array}{l}\text { Patients who had late-stage bilateral osteonecrosis were } \\
\text { randomly treated with bilateral THA }\end{array}$ \\
\hline $\begin{array}{l}\text { Lombardi Jr. et al. } \\
{[12]}\end{array}$ & $\begin{array}{l}\text { Retrospective, } \\
\text { observational } \\
\text { study }\end{array}$ & $131(46 / 85)$ & $\begin{array}{l}52(29-72) / \\
51(22-78)\end{array}$ & $67 / 97$ & Patients who underwent THA using a MHP \\
\hline Mcpherson et al. [36] & $\begin{array}{l}\text { Retrospective, } \\
\text { matched pair }\end{array}$ & $84(42 / 42)$ & $\begin{array}{l}55 \pm 11.4 / \\
56.5 \pm 11.7\end{array}$ & $36 / 48$ & $\begin{array}{l}\text { Patients of the same gender, bone type, activity level, } \\
\text { and diagnosis, ages within } 5 \text { years, weight within } \\
25 \text { pounds, Charnley activity class }\end{array}$ \\
\hline Parvizi et al. [9] & $\begin{array}{l}\text { Prospective, } \\
\text { matched-pair }\end{array}$ & $86(43 / 43)$ & $\begin{array}{l}66.8 \pm 6.2 / \\
65.7 \pm 5.9\end{array}$ & $N R$ & $\begin{array}{l}\text { The patients matched for age, sex, weight, diagnosis, } \\
\text { Charnley class, operative approach, bone quality, } \\
\text { femoral head size, type of acetabular component, } \\
\text { and duration of follow-up }\end{array}$ \\
\hline Paulsen et al. [38] & $\begin{array}{l}\text { Retrospective } \\
\text { comparison }\end{array}$ & $3,158 / 4,749$ & NR & $3,834 / 4,073$ & $\begin{array}{l}\text { Patients underwent primary uncemented THA, who } \\
\text { were younger than } 70 \text { years of age at surgery }\end{array}$ \\
\hline Ranawat et al. [35] & $\mathrm{RCT}$ & $174(92 / 82)$ & $\begin{array}{l}54.9(29.4-67.5) / \\
55.5(28.6-71.8)\end{array}$ & $38 / 114$ & $\begin{array}{l}\text { Patients received cementless THA with the Ranawat-Burstein } \\
\text { metaphyseal-diaphyseal fit hip system }\end{array}$ \\
\hline Rothman et al. [10] & $\begin{array}{l}\text { Retrospective, } \\
\text { matched pair }\end{array}$ & $104(52 / 52)$ & $64(31.2-86.1)$ & $49 / 49$ & $\begin{array}{l}\text { Consecutive THA with use of Taperloc stem, matched } \\
\text { for age, sex, weight, diagnosis, Charnley class, operative } \\
\text { approach, and duration of follow-up }\end{array}$ \\
\hline $\begin{array}{l}\text { Sanchez-Sotelo et al. } \\
\text { [37] }\end{array}$ & $\begin{array}{l}\text { Retrospective, } \\
\text { matched-pair }\end{array}$ & $136(68 / 68)$ & $\begin{array}{l}54(23-66) / \\
56(22-67)\end{array}$ & $56 / 80$ & $\begin{array}{l}\text { Patients who had a primary hip replacement with } \\
\text { insertion of either a porous-coated or HA-coated } \\
\text { Omniflex femoral componene }\end{array}$ \\
\hline Sano et al. [39] & $\begin{array}{l}\text { Retrospective, } \\
\text { observational } \\
\text { study }\end{array}$ & $55(24 / 31)$ & $\begin{array}{l}64.0(51-83) / \\
62.7(41-80)\end{array}$ & $49 / 3$ & $\begin{array}{l}\text { Patients in whom surgery was performed at least } \\
2 \text { years before the present study }\end{array}$ \\
\hline Santori et al. [14] & $\begin{array}{l}\text { Retrospective, } \\
\text { observational } \\
\text { study }\end{array}$ & $227(158 / 69)$ & NR & $N R$ & Patients underwent THA with the anatomic prosthesis \\
\hline Søballe et al. [4] & $\mathrm{RCT}$ & $26(14 / 12)$ & $\begin{array}{l}56.8(48-63) / \\
58.6(50-68)\end{array}$ & $N R$ & $\begin{array}{l}\text { Patients who underwent THR to receive prosthetic } \\
\text { with either Ti-alloy coating or HA coating }\end{array}$ \\
\hline Tanzer et al. [22] & $\mathrm{RCT}$ & $39(17 / 22)$ & $\begin{array}{l}66(54-80) / \\
64(43-78)\end{array}$ & $13 / 26$ & Patients undergoing a cementless THA \\
\hline Tanzer et al. [31] & $\mathrm{RCT}$ & $\begin{array}{l}318(164 / \\
154)\end{array}$ & $\begin{array}{l}64.5 \pm 9.9 / \\
63.1 \pm 10.5\end{array}$ & $153 / 165$ & All patients who underwent cementless THA \\
\hline Yee et al. [30] & $\mathrm{RCT}$ & $62(35 / 27)$ & $\begin{array}{l}48.2 \pm 9.0 / \\
50.4 \pm 8.7\end{array}$ & $11 / 22$ & $\begin{array}{l}\text { Men younger than } 60 \text { years of age and women younger } \\
\text { than } 65 \text { years of age undergoing primary THA }\end{array}$ \\
\hline Yoon et al. [29] & $\mathrm{RCT}$ & $75(37 / 38)$ & $\begin{array}{l}45.3(20-69) / \\
46.0(23-71)\end{array}$ & $14 / 49$ & $\begin{array}{l}\text { Patients who underwent THA use a multilock femoral } \\
\text { stem with or without HA/TCP coating }\end{array}$ \\
\hline
\end{tabular}


Table 2 Details of co-factors and measurement of studies

\begin{tabular}{|c|c|c|c|c|c|c|c|}
\hline Study & $\begin{array}{l}\text { Purity and } \\
\text { thickness of HA }\end{array}$ & Surgery approach & Co-factors & $\begin{array}{l}\text { Follow-up } \\
\text { (years) }\end{array}$ & Missing information & Hip implant & Outcome measurement \\
\hline $\begin{array}{l}\text { Camazzola } \\
\text { et al. [28] }\end{array}$ & NR & Hardinge approach & $\begin{array}{l}\text { Routine antibiotic prophylaxis; } \\
\text { anticoagulation with dicumarin } \\
\text { was used preoperatively and for } \\
\text { a total of } 3 \text { months postoperatively, } \\
\text { full weight-bearing for } 6 \text { weeks }\end{array}$ & $\begin{array}{l}13 \text { years and } \\
5 \text { months } \\
\text { (12-15 years } \\
\text { and } 3 \text { months) }\end{array}$ & $\begin{array}{l}4 \text { patients were lost to follow-up, } 8 \\
\text { died. } 1 \text { refuced to participate in the } \\
\text { questionnaire or clinical follow-up }\end{array}$ & $\begin{array}{l}\text { Mallory-Head } \\
\text { porous stem } \\
\text { (Biomet) }\end{array}$ & $\begin{array}{l}\text { HHS, radiographic outcome, } \\
\text { thigh pain, survival }\end{array}$ \\
\hline Dorr et al. [16] & $\begin{array}{l}94 \% \text { purity, } \\
50-60 \mu \mathrm{m}\end{array}$ & NR & NR & $6.5(5-7.9)$ & No & $\begin{array}{l}\text { Anatomic porous } \\
\text { replacement—I hip } \\
\text { stem (Intermedics } \\
\text { Ortho) }\end{array}$ & HHS, radiographic outcome \\
\hline $\begin{array}{l}\text { Hamadouche } \\
\text { et al. [34] }\end{array}$ & $100 \pm 30 \mu \mathrm{m}$ & $\begin{array}{l}\text { Posterolateral } \\
\text { approach and a } \\
\text { Hardinge lateral } \\
\text { approach }\end{array}$ & $\begin{array}{l}\text { Postoperative management include } \\
\text { administration of systemic antibiotics } \\
\text { for } 48 \mathrm{~h} \text {, preventative anticoagulation } \\
\text { therapy until full weight-bearing, and } \\
\text { NSAID for } 5 \text { days to prevent heterotopic } \\
\text { ossification, partial weight-bearing was } \\
\text { allowed for } 6 \text { weeks followed by full } \\
\text { weight-bearing }\end{array}$ & $9.18(3.93-10.28)$ & $\begin{array}{l}\text { One patient from each group died } \\
\text { from an unrelated cause at three } \\
\text { months and at } 27 \text { months after } \\
\text { surgery, three patients were lost to } \\
\text { follow-up at a mean of three years, } \\
\text { of these, one belonged to the HA } \\
\text { and two to the GB group }\end{array}$ & Profile (DePuy) & HHS, radiographic outcome \\
\hline $\begin{array}{l}\text { Incavo et al. } \\
\text { [32] }\end{array}$ & NR & NR & NR & 4 & no & ProWle (DePuy) & HHS, radiographic outcome \\
\hline Kim et al. [23] & $30 \mu \mathrm{m}$ & $\begin{array}{l}\text { Posterolateral } \\
\text { approach }\end{array}$ & $\begin{array}{l}\text { Stand on the second postoperative day. } \\
\text { Partial weight-bearing with crutches as } \\
\text { tolerated, full weight-bearing was allowed } \\
\text { at } 6 \text { weeks after surgery }\end{array}$ & $6.6(5-7)$ & No & $\begin{array}{l}\text { Cementless IPS } \\
\text { femoral } \\
\text { component } \\
\text { (DePuy) }\end{array}$ & $\begin{array}{l}\text { HHS, functional outcome, } \\
\text { thigh pain, radiographic } \\
\text { outcome, survivorship, } \\
\text { complications }\end{array}$ \\
\hline Kim et al. [24] & $30 \mu \mathrm{m}$ & $\begin{array}{l}\text { Posterolateral } \\
\text { approach }\end{array}$ & NR & $15.6(15-16)$ & 3 were lost to follow-up, 2 died & $\begin{array}{l}\text { IPS femoral stem } \\
\text { (DePuy) }\end{array}$ & $\begin{array}{l}\text { HHS, WOMAC, thigh pain, } \\
\text { Los Angeles activity score, } \\
\text { survivorship }\end{array}$ \\
\hline $\begin{array}{l}\text { Lee and Lee } \\
2007[33]\end{array}$ & $\begin{array}{l}98 \% \text { purity, } \\
150-250 \mu \mathrm{m}\end{array}$ & $\begin{array}{l}\text { Direct lateral } \\
\text { approach } \\
\text { (transgluteal } \\
\text { approach) }\end{array}$ & $\begin{array}{l}\text { Hip joint motion and ambulation using } \\
\text { a wheelchair were allowed from the first } \\
\text { postoperative week, crutch walking with } \\
\text { partial weight-bearing began } 3 \text { to } 4 \text { weeks } \\
\text { after the second operation. In addition, the } \\
\text { patients used a cane for additional } 2 \text { to } \\
4 \text { months until they could walk well } \\
\text { without any support }\end{array}$ & $143(123-168)$ & $\begin{array}{l}4 \text { patients died, } 5 \text { patients were } \\
\text { lost }\end{array}$ & Spotorno (Zimmer) & $\begin{array}{l}\text { Merle d'Aubigne and Postel } \\
\text { score, radiographic outcome }\end{array}$ \\
\hline $\begin{array}{l}\text { Lombardi, Jr. } \\
\text { et al. [12] }\end{array}$ & $\begin{array}{l}95 \% \text { purity, } \\
50-75 \mu \mathrm{m}\end{array}$ & NR & $\begin{array}{l}\text { Routine clinical evaluation was } \\
\text { performed under the supervision } \\
\text { of the operative surgeons }\end{array}$ & $\begin{array}{l}14.5(10.2-16.6) / \\
16.9(11.4-18.5)\end{array}$ & $\begin{array}{l}27 \text { patients in the MHP group and } 5 \\
\text { patients in the MHP HA group were } \\
\text { decreased from causes unrelated to } \\
\text { the index surgery. In addition, } 14 \\
\text { patients ( } 14 \text { hips, } 10.8 \% \text { ) in the MHP } \\
\text { group and nine patients (10 hips, } \\
\text { 16.4\%) in the MHP HA group had not } \\
\text { returned for minimum 10-year follow- } \\
\text { up and were lost to contact }\end{array}$ & $\begin{array}{l}\text { Mallory-Head } \\
\text { porous stem } \\
\text { (Biomet) }\end{array}$ & $\begin{array}{l}\text { HHS, radiographic findings, } \\
\text { survival }\end{array}$ \\
\hline $\begin{array}{l}\text { Mcpherson } \\
\text { et al. [36] }\end{array}$ & $\begin{array}{l}94 \% \text { purity, } \\
50-60 \mu \mathrm{m}\end{array}$ & NR & NR & 3 & No & $\begin{array}{l}\text { Anatomic porous } \\
\text { replacement-I hip } \\
\text { stem (intermedics } \\
\text { orthopedics) }\end{array}$ & $\begin{array}{l}\text { HHS, radiographic fixation } \\
\text { scores }\end{array}$ \\
\hline
\end{tabular}


Table 2 Details of co-factors and measurement of studies (Continued)

\begin{tabular}{|c|c|c|c|c|c|c|c|}
\hline Parvizi et al. [9] & NR & NR & $\mathrm{NR}$ & $9.2 \pm 4.8 / 10.1 \pm 4.6$ & $\begin{array}{l}\text { Each one of the members of nine } \\
\text { pairs of patients is dead }\end{array}$ & $\begin{array}{l}\text { Taperloc, Biomet, } \\
\text { Warsaw, Indiana }\end{array}$ & HHS, radiographic findings \\
\hline $\begin{array}{l}\text { Paulsen et al. } \\
\text { [38] }\end{array}$ & $\begin{array}{l}\text { 95-97\% purity, } \\
50-75 \mu \mathrm{m}\end{array}$ & NR & NR & 3.2 & No & Biometric (Biomet) & Time to implant failure \\
\hline $\begin{array}{l}\text { Ranawat et al. } \\
\text { [35] }\end{array}$ & $\begin{array}{l}95 \% \text { purity, } \\
50-75 \mu \mathrm{m}\end{array}$ & $\begin{array}{l}\text { Posterolateral } \\
\text { approach }\end{array}$ & $\begin{array}{l}\text { Standard postoperative rehabilitation } \\
\text { protocol }\end{array}$ & $\begin{array}{l}5(3-8) \text { for thigh } \\
\text { pain, } 17.7+-0.8 \\
(16.3-20)\end{array}$ & $\begin{array}{l}53 \text { patients were deceased, } 28 \\
\text { patients were lost to follow-up }\end{array}$ & $\begin{array}{l}\text { Ranawat-Burstein } \\
\text { metaphyseal- } \\
\text { diaphyseal fit } \\
\text { femoral stem } \\
\text { (Biomet) }\end{array}$ & $\begin{array}{l}\text { HSS, functional outcome } \\
\text { (patient assessment } \\
\text { questionnaire), incidence of } \\
\text { pain, radiographic outcome, } \\
\text { stem subsidence Kaplan-Meier } \\
\text { survivorship }\end{array}$ \\
\hline $\begin{array}{l}\text { Rothman et al. } \\
\text { [10] }\end{array}$ & $\begin{array}{l}95 \% \text { purity, } \\
50-75 \mu \mathrm{m}\end{array}$ & NR & $\begin{array}{l}\text { Prophylactic antibiotics were given } \\
\text { intravenously at the time of the } \\
\text { operation and were continued for } \\
48 \mathrm{~h} \text {. Ten milligrams of low-dose } \\
\text { warfarin was given on the night } \\
\text { of the operation, instructed to bear } \\
\text { only } 10 \% \text { of the body weight on } \\
\text { the affected limb for } 6 \text { weeks, at } \\
\text { which time, they progressed to use } \\
\text { of a cane }\end{array}$ & $2.2(2-3.4)$ & No & $\begin{array}{l}\text { Taperloc stem } \\
\text { (Biomet) }\end{array}$ & $\begin{array}{l}\text { Charnley scores, radiographic } \\
\text { outcome }\end{array}$ \\
\hline $\begin{array}{l}\text { Sanchez-Sotelo } \\
\text { et al. [37] }\end{array}$ & NR & NR & NR & $\begin{array}{l}6.7(2.4-9.1) / 9.3 \\
(2.2-11.4)\end{array}$ & No & $\begin{array}{l}\text { Omniflex stem } \\
\text { (Osteonics } \\
\text { Corporation) }\end{array}$ & HHS, radiographs, survival \\
\hline Sano et al. [39] & NR & Posterior approach & $\begin{array}{l}\text { Partial weight-bearing was allowed } \\
1 \text { week after the operation, with } \\
\text { full weight-bearing after } 3 \text { weeks }\end{array}$ & $34 / 52 \mathrm{~m}$ & No & $\begin{array}{l}\text { Biomet (Warsaw); } \\
\text { Stryker(Fairfield Rd) }\end{array}$ & $\begin{array}{l}\text { HHS, BMD, radiographic } \\
\text { outcome }\end{array}$ \\
\hline $\begin{array}{l}\text { Santori et al. } \\
{[14]}\end{array}$ & $\begin{array}{l}70 \% \text { purity, } \\
80-130 \mu \mathrm{m}\end{array}$ & NR & $\begin{array}{l}\text { Partial weight bearing with two } \\
\text { canes was allowed on the fifth } \\
\text { postoperative day and progressed } \\
\text { to one cane on day } 30\end{array}$ & 70 m (60-84) & No & $\begin{array}{l}\text { Anatomic prosthesis } \\
\text { (Zimmer) }\end{array}$ & $\begin{array}{l}\text { HHS, thigh pain, radiographic } \\
\text { evaluation }\end{array}$ \\
\hline $\begin{array}{l}\text { Søballe et al. } \\
{[4]}\end{array}$ & $50-75 \mu \mathrm{m}$ & $\begin{array}{l}\text { Posterolateral } \\
\text { approach }\end{array}$ & $\begin{array}{l}\text { Prophylactic antibiotics and anti- } \\
\text { thromboembolic drugs, mobilized } \\
\text { on the third postoperative day and } \\
\text { instructed to walk with protected } \\
\text { weight-bearing for the first six } \\
\text { postoperative weeks }\end{array}$ & 1 & $\begin{array}{l}11 \text { patients were excluded from } \\
\text { RSA because of technical errors, } \\
1 \text { patient with bilateral THR died } \\
\text { from unrelated disease }\end{array}$ & Biometric (Biomet) & $\begin{array}{l}\text { HHS, the visual analog scale } \\
\text { score, radiographs data, RSA }\end{array}$ \\
\hline $\begin{array}{l}\text { Tanzer et al. } \\
\text { [22] }\end{array}$ & $\begin{array}{l}80 \% \mathrm{HA}, 20 \% \\
\text { TCP, } 80 \mu \mathrm{m}\end{array}$ & $\begin{array}{l}\text { Posterolateral } \\
\text { approach }\end{array}$ & $\begin{array}{l}\text { All patients remained non-weight- } \\
\text { bearing for } 6 \text { weeks, followed by } \\
\text { progressive weight-bearing as } \\
\text { tolerated }\end{array}$ & 2 & No & $\begin{array}{l}\text { Cementless } \\
\text { multilock stem } \\
\text { (Zimmer) }\end{array}$ & $\begin{array}{l}\text { HHS, periprosthetic BMD } \\
\text { measurement }\end{array}$ \\
\hline $\begin{array}{l}\text { Tanzer et al. } \\
\text { [31] }\end{array}$ & $\begin{array}{l}80 \% \mathrm{HA}, 20 \% \\
\text { TCP, } 80 \mu \mathrm{m}\end{array}$ & $\begin{array}{l}\text { Posterolateral } \\
\text { approach for } 64 \% \\
\text { and } 69 \% \text { in groups } \\
\text { uncoated and } \\
\text { coated, lateral } \\
\text { approach was used } \\
\text { in the remainder }\end{array}$ & $\begin{array}{l}\text { All patients were non-weight-bearing } \\
\text { for } 6 \text { weeks postoperatively, followed } \\
\text { by progressive weight-bearing as } \\
\text { tolerated }\end{array}$ & 37 m ( $2-5$ years $)$ & $\begin{array}{l}16 \text { patients in the group with } \\
\text { uncoated and } 11 \text { patients in the } \\
\text { group with coated components } \\
\text { withdraw or were lost to follow-up; } \\
4 \text { in uncoated and } 3 \text { in coated died }\end{array}$ & $\begin{array}{l}\text { Cementless } \\
\text { multilock stem } \\
\text { (Zimmer) }\end{array}$ & $\begin{array}{l}\text { HHS, WOMAC, radiographic } \\
\text { data }\end{array}$ \\
\hline
\end{tabular}


Table 2 Details of co-factors and measurement of studies (Continued)

\begin{tabular}{|c|c|c|c|c|c|c|c|}
\hline Yee et al. [30] & $\begin{array}{l}95 \% \text { purity, } \\
50-70 \mu \mathrm{m}\end{array}$ & $\begin{array}{l}\text { A modified lateral } \\
\text { Hardinge approach }\end{array}$ & $\begin{array}{l}\text { Routine prophylactic antibiotic(cefazolin } \\
\text { sodium) was administered before surgery } \\
\text { and } 48 \mathrm{~h} \text { after surgery; anticoagulation with } \\
\text { dicumarin was given: } 5 \mathrm{mg} \text { orally the night } \\
\text { before surgery and daily for a duration of } \\
3 \text { months after surgery. Physical therapy was } \\
\text { commenced on the first or second day after } \\
\text { surgery. Tough weight-bearing with crutches } \\
\text { for } 6 \text { weeks was allowed for uncomplicated } \\
\text { cases. Progression to full weight-bearing as } \\
\text { tolerated was allowed after } 6 \text { weeks }\end{array}$ & $4.6(3-7)$ & $\begin{array}{l}6 \text { patients were lost to follow-up, } 1 \\
\text { died of cardiac causes.1 patient } \\
\text { declined additional participation in } \\
\text { the study after surgery, } 1 \text { with } \\
\text { bilateral THA was involved in a motor } \\
\text { vehicle accident that resulted in a } \\
\text { periprosthetic fracture of one hip }\end{array}$ & $\begin{array}{l}\text { Mallory-Head } \\
\text { porous femoral } \\
\text { stem (Biomet) }\end{array}$ & $\begin{array}{l}\text { HHS, routine radiographs, } \\
\text { survivorship }\end{array}$ \\
\hline Yoon et al. [29] & $\begin{array}{l}70 \% \mathrm{HA}, 30 \% \\
\text { TCP, } 70 \mu \mathrm{m}\end{array}$ & $\begin{array}{l}\text { Hardinge's lateral } \\
\text { approach }\end{array}$ & $\begin{array}{l}\text { Instruted to walk with partial } \\
\text { weight-bearing with the aid of } 2 \\
\text { crutches for } 4 \text { weeks after surgery }\end{array}$ & $\begin{array}{l}127.4 \mathrm{~m}(96-144) / \\
127(108-144)\end{array}$ & $\begin{array}{l}2 \text { patients in the coated group } \\
\text { died of myocardial infarction and } \\
\text { cerebral infarction, } 2 \text { patients in } \\
\text { coated and } 2 \text { in uncoated were } \\
\text { lost to follow-up }\end{array}$ & $\begin{array}{l}\text { Multilock femoral } \\
\text { stem (Zimmer) }\end{array}$ & $\begin{array}{l}\text { HHS, radiographic evaluation, } \\
\text { thigh pain }\end{array}$ \\
\hline
\end{tabular}

HA hydroxyapatite, $T C P$ tricalcium ph
hospital for special surgery hip score 
Table 3 Methodologic quality of included studies

\begin{tabular}{|c|c|c|c|c|c|c|c|c|c|c|c|c|c|}
\hline Study & $\begin{array}{l}\text { Randomized } \\
\text { adequately }^{\mathrm{a}}\end{array}$ & $\begin{array}{l}\text { Allocation } \\
\text { concealed }\end{array}$ & $\begin{array}{l}\text { Similar } \\
\text { baseline }\end{array}$ & $\begin{array}{l}\text { Patient } \\
\text { blinded }\end{array}$ & $\begin{array}{l}\text { Care } \\
\text { provider } \\
\text { blinded }\end{array}$ & $\begin{array}{l}\text { Outcome } \\
\text { assessor } \\
\text { blinded }\end{array}$ & $\begin{array}{l}\text { Avoid } \\
\text { selective } \\
\text { reporting }\end{array}$ & $\begin{array}{l}\text { Similar or } \\
\text { avoided } \\
\text { cofactors }\end{array}$ & $\begin{array}{l}\text { Patients' } \\
\text { compliance }^{\text {b }}\end{array}$ & $\begin{array}{l}\text { Acceptable } \\
\text { drop-out rate }\end{array}$ & $\begin{array}{l}\text { Similar } \\
\text { timing }\end{array}$ & $\begin{array}{l}\text { ITT } \\
\text { analysis }^{d}\end{array}$ & Quality $^{\mathrm{e}}$ \\
\hline Hamadouche et al. [34] & Yes & Unclear & Yes & Unclear & Unclear & Yes & Yes & Yes & Yes & Yes & Yes & No & High \\
\hline Incavo et al. [32] & Yes & Unclear & Yes & Unclear & Unclear & Unclear & Yes & Yes & Yes & Yes & Yes & No & High \\
\hline Kim et al. [23] & Yes & Yes & Yes & Unclear & Unclear & Unclear & Yes & Yes & Yes & Yes & Yes & No & High \\
\hline Kim et al. [24] & Yes & Unclear & Yes & Yes & Unclear & Yes & Yes & Yes & Yes & Yes & Yes & No & High \\
\hline Lee and Lee [33] & Yes & Unclear & Yes & Unclear & Unclear & Unclear & Yes & Yes & Yes & Yes & Yes & No & High \\
\hline Søballe et al. [4] & Yes & Yes & Yes & Unclear & Unclear & Yes & Yes & Yes & Yes & Yes & Yes & No & High \\
\hline Tanzer et al. [22] & Yes & Yes & Yes & Unclear & Unclear & Unclear & Yes & Yes & Yes & Yes & Yes & No & High \\
\hline Tanzer et al. [31] & Yes & Yes & Yes & Yes & Yes & Yes & Yes & Yes & Yes & Yes & Yes & No & High \\
\hline Yee et al. [30] & Yes & Unclear & Yes & Unclear & Unclear & Yes & Yes & Yes & Yes & Yes & Yes & No & High \\
\hline Yoon et al. [29] & Yes & Yes & Yes & Unclear & Unclear & Unclear & Yes & Yes & Yes & Yes & Yes & No & High \\
\hline Camazzola et al. [28] & Yes & Unclear & Yes & Unclear & Unclear & Yes & Yes & Yes & Yes & No & Yes & No & Moderate \\
\hline Dorr et al. [16] & Unclear & Unclear & Yes & Unclear & Unclear & Unclear & Yes & Yes & Yes & Unclear & Yes & Unclear & Moderate \\
\hline Lombardi, Jr. et al. [12] & Unclear & Unclear & Yes & Unclear & Unclear & Unclear & Yes & Yes & Yes & No & Yes & No & Moderate \\
\hline Mcpherson et al. [36] & Unclear & Unclear & Yes & Yes & Unclear & Unclear & Yes & Yes & Yes & Yes & Yes & No & Moderate \\
\hline Parvizi et al. [9] & Unclear & Unclear & Yes & Unclear & Unclear & Unclear & Yes & Yes & Yes & Yes & Yes & No & Moderate \\
\hline Paulsen et al. [38] & Unclear & Unclear & Yes & Unclear & Unclear & Unclear & Yes & Yes & Yes & Yes & Yes & No & Moderate \\
\hline Ranawat et al. [35] & Yes & Unclear & Yes & Unclear & Unclear & Unclear & Yes & Yes & Yes & No & Yes & No & Moderate \\
\hline Rothman et al. [10] & No & Unclear & Yes & No & No & Yes & Yes & Yes & Yes & Yes & Yes & No & Moderate \\
\hline Sanchez-Sotelo et al. [37] & Unclear & Unclear & Yes & Unclear & Unclear & Unclear & Yes & Yesc & Yes & Yes & Yes & No & Moderate \\
\hline Sano et al. [39] & Unclear & Unclear & Yes & Unclear & Unclear & Unclear & Yes & Yes & Yes & Yes & Yes & No & Moderate \\
\hline Santori et al. [14] & Unclear & Unclear & Yes & Unclear & Unclear & Unclear & Yes & Yes & Yes & Yes & Yes & No & Moderate \\
\hline
\end{tabular}

"Only if the method of sequence generated was explicitly described could get a "Yes"; sequence generated by "Dates of Admission" or "Patients Number" received a "No".

"Intermittent treatment or therapy duration less than 6 months means "Yes", otherwise "No".

"Drop-out rate $\geq 20 \%$ means " $\mathrm{No}$ ", otherwise "Yes".

dITT intention-to-treat, only if all randomized patients are analyzed in the group they were allocated to could receive a "Yes".

"The frequency of "Yes" as 7 or greater means "High", greater than 4 but less means "Moderate", 4 or less means "Low". 


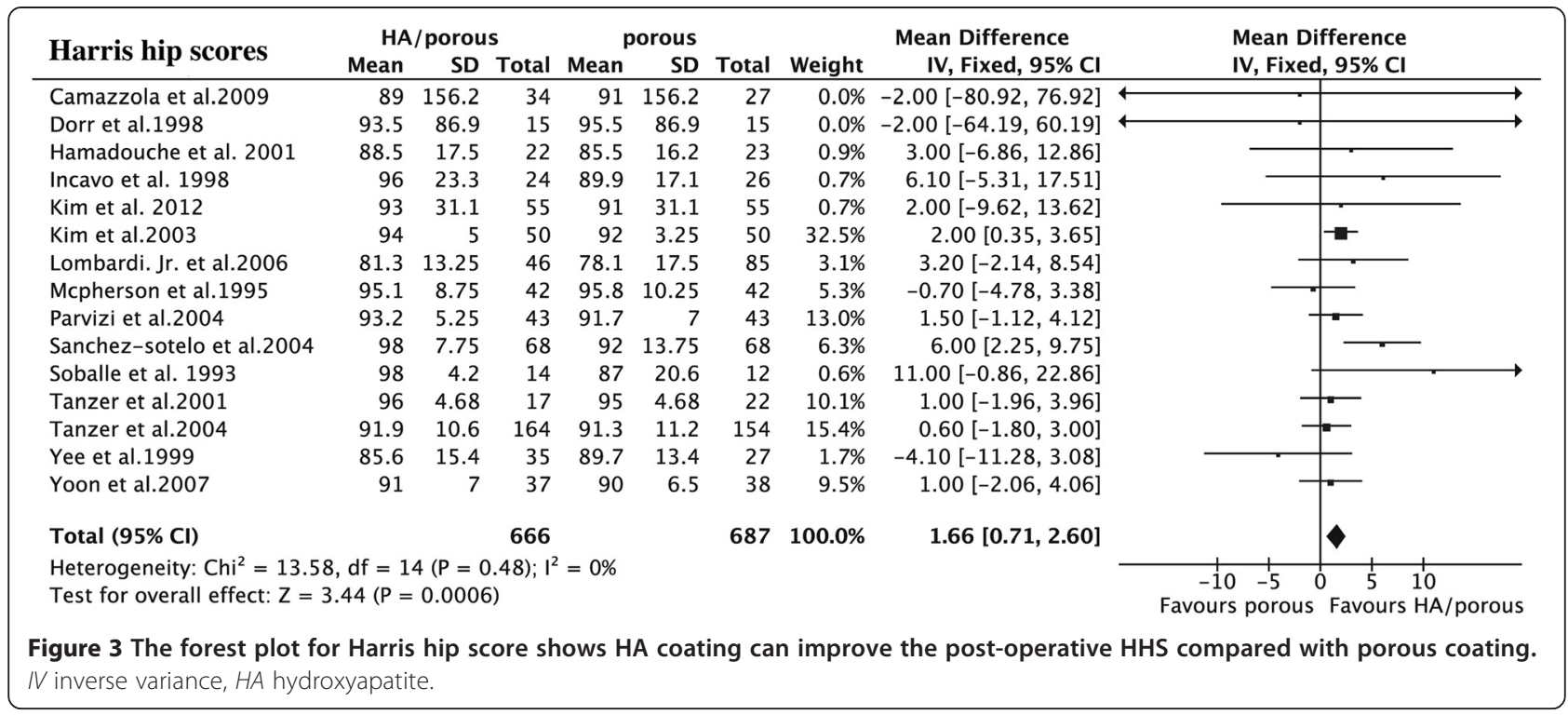

duration was chosen to be the cutoff follow-up duration as there was sufficient data available allowing for the subgroup analysis. Sensitivity analysis was performed through omitting trials to assess the changes in overall effect. Funnel plots were used to assess publication bias among the included trials graphically. Bias can be seen if the plots were widely skewed versus a plot resembling an inverted triangle which represents no bias [27].

\section{Results}

The literature search initially yielded 878 relevant trials. Two of us (CYL and SMM) reviewed the titles and abstracts of all reviews including two of hand-searched. Twelve RCTs [4,22-24,28-35] and nine comparative observation trials $[9,10,12,14,16,36-39]$ were included after applying our eligibility criteria (Figure 1). Funnel plots indicated no publication bias (Figure 2). We recorded the characteristics of 21 trials that were included (Table 1) and details of co-factors and measurement (Table 2). All studies reported a minimum 1 year (median 7.5 years, range 1 to 17.7). We assessed the quality of included trials with the 12-item scale (Table 3). However the intentionto-treat analysis was rarely reported, and no outcome was selectively reported in all studies.

The results showed that the HA presented higher HHS than the porous group ( 15 trials, $N=1,353$, WMD = $1.66,95 \%$ CI 0.71 to $2.60, p=0.0006$ ) (Figure 3), could decrease the thigh pain incidence ( 6 trials, $N=724$, $\mathrm{OR}=$ $0.53,95 \% \mathrm{CI} 0.32$ to $0.87, p=0.01$ ) (Figure 4 ), and had less femoral osteolysis ( 5 trials, $N=386$, OR $=0.52$, 95\% CI 0.31 to $0.86, p=0.01$ ) (Figure 5), while there was no difference in the survivorship from aseptic loosening (16 trials, $N=$ 9,472, $\mathrm{RR}=1.00,95 \% \mathrm{CI} 1.00$ to $1.00, p=0.32$ ) (Figure 6), polyethylene wear ( 4 trials, $N=347$, WMD $=-0.02$, $95 \%$ CI -0.04 to $0.00, p=0.08$ ) (Figure 7) and radiolucent lines (6 trials, $N=566$, OR $=0.95,95 \%$ CI 0.67 to $1.35, p=0.78$ ) ( Figure 8 ) between the two groups.

There were no heterogeneities for HHS $\left(I^{2}=0 \%\right)$, survivorship from the aseptic loosening $\left(I^{2}=0 \%\right)$, radiolucent lines $\left(I^{2}=0 \%\right)$, or femoral osteolysis $\left(I^{2}=46 \%\right)$. The results

\begin{tabular}{|c|c|c|c|c|c|c|c|c|c|}
\hline \multirow{2}{*}{$\begin{array}{l}\text { Thigh pain } \\
\text { Kim et al.2003 }\end{array}$} & \multicolumn{2}{|c|}{ HA/porous } & \multicolumn{2}{|c|}{ porous } & Weight & $\begin{array}{c}\text { Odds Ratio } \\
\text { M-H, Fixed, 95\% Cl }\end{array}$ & \multicolumn{2}{|r|}{$\begin{array}{c}\text { Odds Ratio } \\
\text { M-H, Fixed, 95\% CI }\end{array}$} & \\
\hline & 2 & 50 & 2 & 50 & $4.4 \%$ & $1.00[0.14,7.39]$ & & & \\
\hline Parvizi et al.2004 & 1 & 43 & 1 & 43 & $2.2 \%$ & $1.00[0.06,16.52]$ & & & \\
\hline Ranawat et al. 2011 & 11 & 92 & 20 & 82 & $42.5 \%$ & $0.42[0.19,0.94]$ & & & \\
\hline Santori et al.2001 & 2 & 158 & 10 & 69 & $31.4 \%$ & $0.08[0.02,0.36]$ & & & \\
\hline Yee et al.1999 & 15 & 35 & 9 & 27 & $13.3 \%$ & $1.50[0.53,4.26]$ & & - & \\
\hline Yoon et al.2007 & 3 & 37 & 3 & 38 & $6.2 \%$ & $1.03[0.19,5.46]$ & & & \\
\hline Total $(95 \% \mathrm{Cl})$ & & 415 & & 309 & $100.0 \%$ & $0.53[0.32,0.87]$ & & & \\
\hline Total events & 34 & & 45 & & & & & & \\
\hline $\begin{array}{l}\text { Heterogeneity: } \mathrm{Chi}^{2} \\
\text { Test for overall effec }\end{array}$ & $\begin{array}{l}11.40, d \\
Z=2.51\end{array}$ & $\begin{array}{l}f=5(P \\
(P=0\end{array}$ & $\begin{array}{l}=0.04) \\
.01)\end{array}$ & $; 1^{2}=$ & & & $\begin{array}{r}0.005 \\
\mathrm{Fav}\end{array}$ & $\begin{array}{llr}0.1 & 1 & 10 \\
\text { rs porous } & \text { Favours }\end{array}$ & $\begin{array}{l}200 \\
\text { oorous }\end{array}$ \\
\hline
\end{tabular}

Figure 4 The forest plot for thigh pain incidence shows that HA coating can reduce it compared with porous coating. HA hydroxyapatite. 


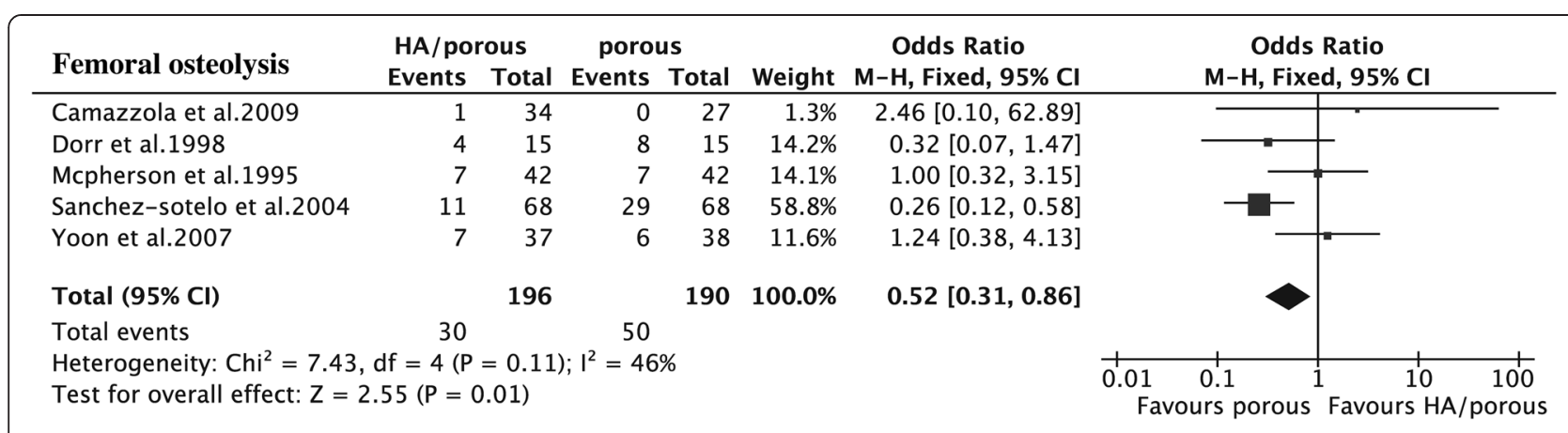

Figure $\mathbf{5}$ The forest plot for femoral osteolysis shows that HA coating has less osteolysis compared with porous coating. HA hydroxyapatite.

of thigh pain incidence and polyethylene wear was heterogeneous in some degree $\left(I^{2}=56 \% ; I^{2}=96 \%\right)$. The heterogeneity of thigh pain incidence can not be explained by the study design, quality of study, thickness of HA, implant design or follow-up duration maybe because of the potential co-factors, such as weight-bearing after the operation. As to the limitation of this analysis, we could not set this subgroup analysis. The polyethylene wear can not be explained by purity of HA and duration of follow-up. But when we classified the polyethylene wear into subgroups by the thickness of HA (50-80 $\mu \mathrm{m}$ and $>80 \mu \mathrm{m}$ or $<50 \mu \mathrm{m})$ and implant design (anatomic and non-anatomic), the heterogeneity could no longer be observed $\left(I^{2}=0 \%\right)$.

Subgroup analysis for HHS, survival of implant from aseptic loosening, and radiolucent lines indicated that the included non-RCTs did not affect the RCTs $(p=$ $0.42 ; p=0.27 ; p=0.98 ; p=0.52$ ), while as to incidence of thigh pain and femoral osteolysis, there was a slight difference $(p=0.02 ; p=0.05)$. Our results showed that the longer duration of follow-up tends to have higher HHS $(p=0.11)$ than the shorter one $(\mathrm{WMD}=2.21,95 \% \mathrm{CI}$ 1.05 to 3.37 for duration of follow-up $>6$ years; WMD $=$ $0.58,95 \% \mathrm{CI}-1.04$ to 2.20 for duration $<6$ years) and likewise for the survival $(p=0.11, \mathrm{RR}=1.0195 \%$ CI 0.99 to 1.03 for duration of follow-up $>6$ years, $R R=1.00$, 95\% CI 0.99 to 1.00 for duration of follow-up $<6$ years). The thickness of HA was larger than $80 \mu \mathrm{m}$ or less than $50 \mu \mathrm{m}$, the purity less than $90 \%$, and the anatomic implant reduced incidence of thigh pain and the duration of follow-up did not affect it. It is doubtful that the RCTs and high-quality study had the high incidence of thigh pain and femoral osteolysis (Table 4). The overall result was not significantly altered by omitting trials with a sample sizes less than 70 or those with imputed data.

\section{Discussion}

The primary finding is that HA coating could improve the postoperative HHS, reduce the incidence of thigh pain,

\begin{tabular}{|c|c|c|c|c|c|c|c|}
\hline Survival & \multicolumn{2}{|c|}{ HA/porous } & \multicolumn{2}{|c|}{ porous } & Weight & $\begin{array}{c}\text { Risk Ratio } \\
\text { M-H, Fixed, 95\% CI }\end{array}$ & $\begin{array}{c}\text { Risk Ratio } \\
\text { M-H, Fixed, 95\% Cl }\end{array}$ \\
\hline Camazzola et al.2009 & 34 & 34 & 27 & 27 & $0.7 \%$ & $1.00[0.94,1.07]$ & - \\
\hline Hamadouche et al. 2001 & 21 & 22 & 19 & 23 & $0.4 \%$ & $1.16[0.94,1.42]$ & \\
\hline Kim et al. 2012 & 55 & 55 & 55 & 55 & $1.2 \%$ & $1.00[0.97,1.04]$ & - \\
\hline Kim et al.2003 & 50 & 50 & 50 & 50 & $1.1 \%$ & $1.00[0.96,1.04]$ & - \\
\hline Lee et al. 2007 & 20 & 20 & 20 & 20 & $0.4 \%$ & $1.00[0.91,1.10]$ & 一 \\
\hline Lombardi. Jr. et al.2006 & 46 & 46 & 84 & 85 & $1.3 \%$ & $1.01[0.97,1.05]$ & - \\
\hline Mcpherson et al.1995 & 42 & 42 & 42 & 42 & $0.9 \%$ & $1.00[0.96,1.05]$ & 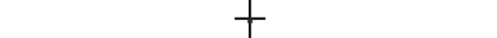 \\
\hline Parvizi et al.2004 & 43 & 43 & 43 & 43 & $1.0 \%$ & $1.00[0.96,1.05]$ & \\
\hline Paulsen et al. 2007 & 3144 & 3158 & 4743 & 4749 & $83.0 \%$ & $1.00[0.99,1.00]$ & \\
\hline Ranawat et al. 2011 & 92 & 92 & 82 & 82 & $1.9 \%$ & $1.00[0.98,1.02]$ & \\
\hline Rothman et al.1996 & 52 & 52 & 52 & 52 & $1.2 \%$ & $1.00[0.96,1.04]$ & - \\
\hline Sanchez-sotelo et al.2004 & 67 & 68 & 65 & 68 & $1.4 \%$ & $1.03[0.97,1.09]$ & - \\
\hline Tanzer et al.2001 & 17 & 17 & 22 & 22 & $0.4 \%$ & $1.00[0.91,1.10]$ & \\
\hline Tanzer et al.2004 & 164 & 164 & 154 & 154 & $3.5 \%$ & $1.00[0.99,1.01]$ & \\
\hline Yee et al.1999 & 35 & 35 & 27 & 27 & $0.7 \%$ & $1.00[0.94,1.07]$ & . \\
\hline Yoon et al.2007 & 37 & 37 & 38 & 38 & $0.8 \%$ & $1.00[0.95,1.05]$ & 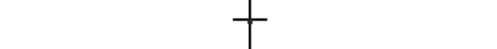 \\
\hline Total $(95 \% \mathrm{Cl})$ & & 3935 & & 5537 & $100.0 \%$ & $1.00[1.00,1.00]$ & \\
\hline Total events & 3919 & & 5523 & & & & \\
\hline $\begin{array}{l}\text { Heterogeneity: } \mathrm{Chi}^{2}=5.01 \\
\text { Test for overall effect: } Z=\end{array}$ & $\begin{array}{l}\mathrm{df}=15(P \\
.99(P=0\end{array}$ & $\begin{array}{l}P=0.99 \\
.32)\end{array}$ & 9); $I^{2}=0$ & & & & 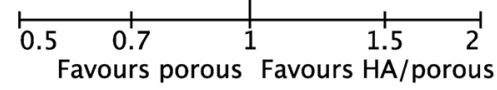 \\
\hline
\end{tabular}




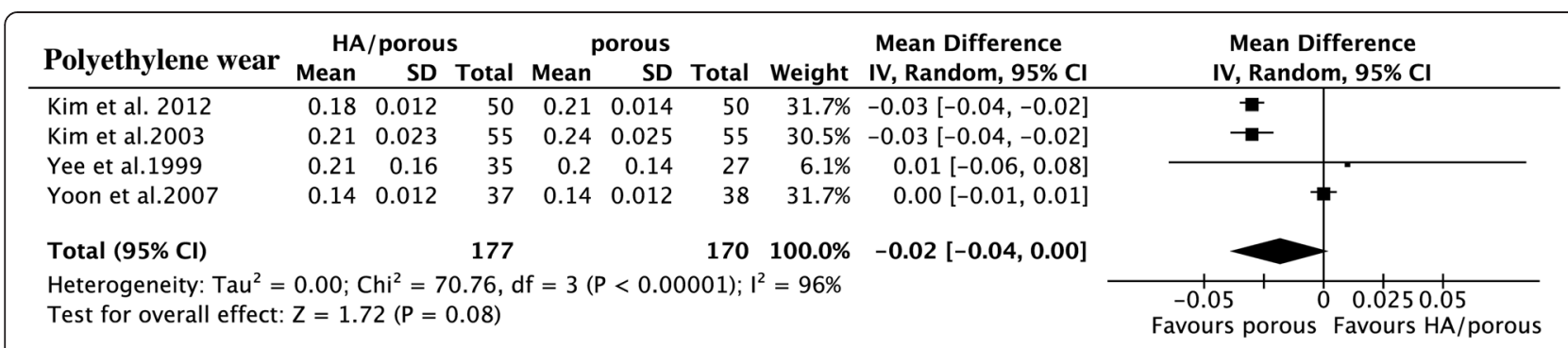

Figure 7 The forest plot for polyethylene wear shows HA coating has less wear compared with porous coating. IV inverse variance, HA hydroxyapatite.

and reduce the incidence of femoral osteolysis while there was no statistical difference of femoral stem survivorship from aseptic loosening, polyethylene wear, and radiolucent lines between the two groups. In addition, the subgroup analyses found that HHS tends to improve in the longer duration of follow-up and so was the prosthetic survival. The longer duration of follow-up, the better advantage of HA coating over porous coating for the HHS and survivorship from aseptic loosening.

In this meta-analysis, we asked: (1) which coating is better with regard to the clinical and radiologic measurements and (2) which modifying factors affect the comparative effect between both coatings.

To the best of our knowledge, the present meta-analysis is the first to comprise all the available comparative observational evidence and to comprehensively investigate the difference in HHS and survivorship and radiographic outcomes between HA and porous coating for THA. As the previous systematic review only included four RCTs of nine studies, one of the included studies did not show clear HHS data and the HA stem was grit-blasted, while porous stem was not. Another previous systematic review did not include new studies published in the later years. We included 12 RCTs and 9 comparison observation studies and developed explicit inclusion and exclusion criteria. Our analysis quintupled the sample size compared with previous meta-analysis $(9,860$ versus 1,764$)$ and had longer duration of follow-up (7.5 years versus 6.5 years). We performed a comprehensive set of subgroup analyses and a sensitivity analysis not only to explain the heterogeneity but also to provide additional insights into the potential influential factors of HHS, survival, thigh pain, and other radiographic outcome.

Our meta-analysis has some limitations. (1) There was variability in the selection criteria of individual trials, including the primary disease, gender, ages of patients, and the type of prosthesis. (2) due to the limited number of included trials, we could not analyze the influence of other clinically relevant factors, such as complications of THA, BMD, and WOMAC osteoarthritis index. (3) Missing information such as declining participation and crossover led to incomplete data and potentially bias. (4) The small sample size in the subgroup analysis reduced the precision of the pooled estimates and the ability to detect the statistical significance of some variables, that is, polyethylene wear. More RCTs would be warranted to clarify them. (5) With the limitation of included studies, we can not analyze the effect of implant design, which needs more study to assess.

One of the most significant results of our analysis is that HA coating had higher HHS and less incidence of thigh pain. The advantage of an HA coating includes superior proximal femoral osteointegration and better preservation of periprosthetic bone quality. The patients with

\begin{tabular}{|c|c|c|c|c|c|c|c|}
\hline Radiolucent lines & \multicolumn{2}{|c|}{ HA/porous } & \multicolumn{2}{|c|}{ porous } & Weight & \multirow{2}{*}{$\begin{array}{c}\text { Odds Ratio } \\
\text { M-H, Fixed, 95\% Cl } \\
0.32[0.07,1.47]\end{array}$} & $\begin{array}{c}\text { Odds Ratio } \\
\text { M-H, Fixed, 95\% Cl }\end{array}$ \\
\hline Dorr et al.1998 & 4 & 15 & 8 & 15 & $9.0 \%$ & & \\
\hline Sano et al. 2008 & 14 & 24 & 14 & 31 & $7.8 \%$ & $1.70[0.58,4.99]$ & \\
\hline Soballe et al. 1993 & 4 & 14 & 4 & 12 & $4.7 \%$ & $0.80[0.15,4.24]$ & \\
\hline Tanzer et al.2004 & 85 & 164 & 82 & 154 & $62.7 \%$ & $0.94[0.61,1.47]$ & \\
\hline Yee et al.1999 & 30 & 35 & 22 & 27 & $5.5 \%$ & $1.36[0.35,5.29]$ & \\
\hline Yoon et al.2007 & 29 & 37 & 31 & 38 & $10.2 \%$ & $0.82[0.26,2.54]$ & \\
\hline Total $(95 \% \mathrm{Cl})$ & & 289 & & 277 & $100.0 \%$ & $0.95[0.67,1.35]$ & \\
\hline Total events & 166 & & 161 & & & & \\
\hline $\begin{array}{l}\text { Heterogeneity: } \mathrm{Chi}^{2}= \\
\text { Test for overall effect }\end{array}$ & $\begin{array}{l}3.47, \mathrm{df} \\
\mathrm{Z}=0.29\end{array}$ & $\begin{array}{l}=5(P= \\
(P=0\end{array}$ & $\begin{array}{l}=0.63) ; 1 \\
.78)\end{array}$ & ${ }^{2}=0 \%$ & & & \begin{tabular}{ccccccc}
1 & 1 & 1 & 1 & 1 & 1 \\
0.1 & 0.2 & 0.5 & 1 & 2 & 5 & 10 \\
\multicolumn{3}{l}{ Favours porous } & Favours & HA/porous
\end{tabular} \\
\hline
\end{tabular}


Table 4 Subgroup analysis of the included studies by different influential factors

\begin{tabular}{|c|c|c|c|c|c|c|c|c|c|c|c|c|}
\hline \multirow[t]{2}{*}{ Factors } & \multicolumn{2}{|c|}{ Harris hip score } & \multicolumn{2}{|l|}{ Survival } & \multicolumn{2}{|c|}{ Incidence of thigh pain } & \multicolumn{2}{|c|}{ Radiolucent lines } & \multicolumn{2}{|l|}{ Femoral osteolysis } & \multicolumn{2}{|c|}{ Polyethylene wear } \\
\hline & $\begin{array}{l}\text { Subgroups } \\
\text { (numbers) }\end{array}$ & $\begin{array}{l}\text { WMD }(95 \% \\
\mathrm{Cl})\end{array}$ & $\begin{array}{l}\text { Subgroups } \\
\text { (numbers) }\end{array}$ & RR $(95 \% \mathrm{Cl})$ & $\begin{array}{l}\text { Subgroups } \\
\text { (numbers) }\end{array}$ & OR $(95 \% \mathrm{Cl})$ & $\begin{array}{l}\text { Subgroups } \\
\text { (numbers) }\end{array}$ & OR $(95 \% \mathrm{Cl})$ & $\begin{array}{l}\text { Subgroups } \\
\text { (numbers) }\end{array}$ & OR $(95 \% \mathrm{Cl})$ & $\begin{array}{l}\text { Subgroups } \\
\text { (numbers) }\end{array}$ & $\begin{array}{l}\text { WMD } \\
(95 \% \mathrm{Cl})\end{array}$ \\
\hline \multirow[t]{3}{*}{$\begin{array}{l}\text { Study } \\
\text { design }\end{array}$} & RCT (10) & $\begin{array}{l}1.41(0.30, \\
2.52)\end{array}$ & $\mathrm{RCT}(10)$ & $\begin{array}{l}1.01 \\
(0.99,1.02)\end{array}$ & RCT (4) & $\begin{array}{l}0.73(0.42 \\
1.28)\end{array}$ & $\mathrm{RCT}(4)$ & $\begin{array}{l}0.95(0.65 \\
1.39)\end{array}$ & $\mathrm{RCT}(2)$ & $\begin{array}{l}1.37 \\
(0.45,4.17)\end{array}$ & $\mathrm{RCT}(4)$ & N.A. \\
\hline & Non-RCT (5) & $\begin{array}{l}2.29(0.50 \\
4.07)\end{array}$ & Non-RCT (6) & $\begin{array}{l}1.00(0.99 \\
1.00)\end{array}$ & Non-RCT (2) & $\begin{array}{l}0.14(0.04 \\
0.49)\end{array}$ & Non-RCT (2) & $\begin{array}{l}0.96(0.41 \\
2.24)\end{array}$ & Non-RCT (3) & $\begin{array}{l}0.39(0.22 \\
0.70)\end{array}$ & Non-RCT (0) & \\
\hline & & $p=0.42$ & & $p=0.27$ & & $p=0.02$ & & $p=0.98$ & & $p=0.05$ & & \\
\hline \multirow[t]{3}{*}{$\begin{array}{l}\text { Study } \\
\text { quality }\end{array}$} & High (9) & $\begin{array}{l}1.41(0.30 \\
2.52)\end{array}$ & High (8) & $\begin{array}{l}1.01(0.99 \\
1.02)\end{array}$ & High (3) & $\begin{array}{l}1.29(0.57 \\
2.88)\end{array}$ & High (4) & $\begin{array}{l}0.95(0.65 \\
1.39)\end{array}$ & High (2) & $\begin{array}{l}1.24(0.38 \\
4.13)\end{array}$ & High (4) & N.A. \\
\hline & Moderate (6) & $\begin{array}{l}2.28(0.50 \\
4.07)\end{array}$ & Moderate (8) & $\begin{array}{l}1.00(0.99 \\
1.00)\end{array}$ & Moderate (3) & $\begin{array}{l}0.30(0.15 \\
0.57)\end{array}$ & Moderate (2) & $\begin{array}{l}0.96(0.41 \\
2.24)\end{array}$ & Moderate (2) & $\begin{array}{l}0.42(0.24 \\
0.74)\end{array}$ & Moderate (0) & \\
\hline & & $p=0.42$ & & $p=0.27$ & & $p=0.006$ & & $p=0.98$ & & $p=0.11$ & & \\
\hline \multirow[t]{3}{*}{$\begin{array}{l}\text { Thickness } \\
\text { of HA }\end{array}$} & 50-80 بm (8) & $\begin{array}{l}0.76(-0.63, \\
2.15)\end{array}$ & $50-80 \mu \mathrm{m}(10)$ & $\begin{array}{l}1.00(0.99 \\
1.00)\end{array}$ & $50-80 \mu m(3)$ & $\begin{array}{l}0.71(0.40 \\
1.27)\end{array}$ & $50-80 \mu \mathrm{m}(5)$ & N.A. & $50-80 \mu \mathrm{m}(3)$ & N.A. & $50-80 \mu \mathrm{m}(2)$ & $\begin{array}{l}0.00 \\
(-0.01,0.01)\end{array}$ \\
\hline & $\begin{array}{l}<50 \mu \mathrm{m} \text { or } \\
>80 \mu \mathrm{m}(3)\end{array}$ & $\begin{array}{l}2.03(0.41 \\
3.64)\end{array}$ & $\begin{array}{l}<50 \mu \mathrm{m} \text { or } \\
>80 \mu \mathrm{m}(4)\end{array}$ & $\begin{array}{l}1.02(0.98, \\
1.06)\end{array}$ & $\begin{array}{l}<50 \mu \mathrm{m} \text { or } \\
>80 \mu \mathrm{m}(2)\end{array}$ & $\begin{array}{l}0.19(0.06, \\
0.58)\end{array}$ & $\begin{array}{l}<50 \mu \mathrm{m} \text { or } \\
>80 \mu \mathrm{m}(0)\end{array}$ & & $\begin{array}{l}<50 \mu m \text { or }>80 \mu m \\
\text { (0) }\end{array}$ & & $\begin{array}{l}<50 \mu \mathrm{m} \text { or } \\
>80 \mu \mathrm{m}(2)\end{array}$ & $\begin{array}{l}-0.03 \\
(-0.03,-0.03)\end{array}$ \\
\hline & & $p=0.24$ & & $p=0.23$ & & $p=0.04$ & & & & & & $p<0.00001$ \\
\hline \multirow[t]{3}{*}{$\begin{array}{l}\text { Purity of } \\
\text { HA }\end{array}$} & $>90 \%(4)$ & $\begin{array}{l}-0.09(-3.04 \\
2.86)\end{array}$ & $>90 \%(7)$ & $\begin{array}{l}1.00(0.99 \\
1.00)\end{array}$ & $>90 \%(2)$ & $\begin{array}{l}0.68(0.36 \\
1.26)\end{array}$ & $>90 \%(3)$ & $\begin{array}{l}0.73(0.31 \\
1.71)\end{array}$ & $>90 \%(2)$ & $\begin{array}{l}0.66(0.27 \\
1.62)\end{array}$ & $>90 \%(1)$ & $\begin{array}{l}0.01 \\
(-0.06,0.08)\end{array}$ \\
\hline & $<90 \%(3)$ & $\begin{array}{l}0.82(-0.77 \\
2.42)\end{array}$ & $<90 \%(3)$ & $\begin{array}{l}1.00(0.98 \\
1.02)\end{array}$ & $<90 \%(3)$ & $\begin{array}{l}0.23(0.08 \\
0.65)\end{array}$ & $<90 \%(2)$ & $\begin{array}{l}0.93(0.61 \\
1.40)\end{array}$ & $<90 \%(1)$ & $\begin{array}{l}1.24(0.38 \\
4.13)\end{array}$ & $<90 \%(1)$ & $\begin{array}{l}0.00 \\
(-0.01,0.01)\end{array}$ \\
\hline & & $p=0.59$ & & $p=0.72$ & & $p=0.08$ & & $p=0.63$ & & $p=0.4$ & & $p=0.79$ \\
\hline \multirow[t]{3}{*}{$\begin{array}{l}\text { Implant } \\
\text { design }\end{array}$} & Anatomic (5) & $\begin{array}{l}1.65(0.15 \\
3.16)\end{array}$ & Anatomic (4) & $\begin{array}{l}1.02(0.99 \\
1.05)\end{array}$ & Anatomic (2) & $\begin{array}{l}0.19(0.06 \\
0.58)\end{array}$ & Anatomic (1) & $\begin{array}{l}0.32(0.07 \\
1.47\end{array}$ & Anatomic (2) & $\begin{array}{l}0.66(0.27 \\
1.62)\end{array}$ & Anatomic (2) & $\begin{array}{l}-0.03 \\
(-0.03,-0.03)\end{array}$ \\
\hline & $\begin{array}{l}\text { Non-anatomic } \\
\text { (10) }\end{array}$ & $\begin{array}{l}1.66(0.44 \\
2.87)\end{array}$ & $\begin{array}{l}\text { Non-anatomic } \\
\text { (12) }\end{array}$ & $\begin{array}{l}1.00(1.00 \\
1.00)\end{array}$ & $\begin{array}{l}\text { Non-anatomic } \\
\text { (4) }\end{array}$ & $\begin{array}{l}0.72(0.41 \\
1.28)\end{array}$ & $\begin{array}{l}\text { Non-anatomic } \\
\text { (5) }\end{array}$ & $\begin{array}{l}1.01(0.71 \\
1.45)\end{array}$ & Non-anatomic (3) & $\begin{array}{l}0.46(0.25 \\
0.85\end{array}$ & $\begin{array}{l}\text { Non-anatomic } \\
\text { (2) }\end{array}$ & $\begin{array}{l}0.00 \\
(-0.01,0.01)\end{array}$ \\
\hline & & $p=1.00$ & & $p=0.24$ & & $p=0.04$ & & $p=0.15$ & & $p=0.52$ & & $p<0.00001$ \\
\hline \multirow[t]{3}{*}{$\begin{array}{l}\text { Follow-up } \\
\text { duration }\end{array}$} & $>6$ years $(9)$ & $\begin{array}{l}2.21(1.05 \\
3.37)\end{array}$ & $>6$ years $(10)$ & $\begin{array}{l}1.01(0.99 \\
1.03)\end{array}$ & $>6$ years $(4)$ & $\begin{array}{l}0.56(0.29 \\
1.08)\end{array}$ & $>6$ years $(2)$ & $\begin{array}{l}0.58(0.24 \\
1.43)\end{array}$ & $>6$ years $(4)$ & $\begin{array}{l}0.44(0.24 \\
0.77)\end{array}$ & $>6$ years $(3)$ & $\begin{array}{l}-0.02 \\
(-0.02,-0.01)\end{array}$ \\
\hline & $<6$ years $(6)$ & $\begin{array}{l}0.58(-1.04 \\
2.20)\end{array}$ & $<6$ years $(6)$ & $\begin{array}{l}1.00(0.99 \\
1.00)\end{array}$ & $<6$ years $(2)$ & $\begin{array}{l}0.50(0.24 \\
1.05)\end{array}$ & $<6$ years $(4)$ & $\begin{array}{l}1.04(0.71 \\
1.52)\end{array}$ & $<6$ years $(1)$ & $\begin{array}{l}1.00(0.32, \\
3.15)\end{array}$ & $<6$ years $(1)$ & $\begin{array}{l}0.01 \\
(-0.06,0.08)\end{array}$ \\
\hline & & $p=0.11$ & & $p=0.11$ & & $p=0.82$ & & $p=0.25$ & & $p=0.2$ & & $p=0.46$ \\
\hline
\end{tabular}

Study design and study quality would affect the incidence of thigh pain and study design has influence on femoral osteolysis. When the thickness of HA is $<50$ or $>80 \mu \mathrm{m}$, it has less thigh pain incidence and polyethylene wear. The anatomic implant has less incidence of thigh pain and polyethylene wear.

WMD weighted mean difference, N.A. not available. HA hydroxyapatite. 
HA-coated stems demonstrated significantly lower incidence of activity-related trochanteric and thigh pain [35]. The thickness and purity of HA and implant design could affect the incidence of thigh pain. The incidence decreased abruptly after the first postoperative year [40]. Thigh pain following uncemented hip arthroplasty was generally transient and would disappear over time.

Femoral osteolysis and polyethylene wear could result to gradual subsidence or loosening of implant. Some studies showed that HA coating had less osteolysis and polyethylene wear $[37,16]$, but in Almeida's study, with the use of HA coated stem, they still found $38 \%$ of osteolysis and $41 \%$ of polyethylene wear in the hips. Their stems were mostly used in young patients, who had greater activity compared to the older patients which might have been the affecting factor. From the retrieved specimen, some studies found that HA coating could increase the amount of ingrowth and attachment of bone leading to the enhanced biological fixation [41]. Moreover, HA-coated Ti implants can achieve a much higher degree of bone apposition and mechanical stability compared to the implants without such a coating [26]. The heterogeneity of polyethylene wear can not be explained by purity of HA and duration of follow-up. But when we classified the polyethylene wear into subgroups by the thickness of HA $(50-80 \mu \mathrm{m}$ and $>80 \mu \mathrm{m}$ or $<50 \mu \mathrm{m})$ and implant design (anatomic and non-anatomic), the heterogeneity could no longer be observed.

The geometry design of the implant has a large impact on the clinical outcome. Joshi's study explored the hypothesis that through redesign, a total hip prosthesis could be developed to substantially reduce stress shielding, then reduce the loosening of the prosthesis [42]. DopicoGonzález assessed effects of implant design geometry by probabilistic finite element tool, she thought the geometry of the implant design clearly affected the sensitivities of maximum nodal micromotion [43]. As the limitation of included studies, we just divided the implant into anatomic and non-anatomic. It needs more studies to assess the effect of implant design. Our study showed that anatomic implant had less incidence of thigh pain and reduced polyethylene wear. Ando et al. found that FMSanatomic stem reduced the proximal stem-bone relative motion and transferred more load to the proximal femur compared to conventional symmetric stems and the FMS, which resulted in better biomechanical stability at least in the early postoperative period [44]. Another study showed an excellent clinical outcome and $98.3 \%$ survival of ABG II implant with HA coating [45]. In Cao's study, they also thought that an anatomically designed prosthesis can provide good clinical results, with low incidence of thigh pain and loosening of the component [46].

$\mathrm{HA}$ is biocompatible and osteoconductive and in contact with bone often develops a mechanically tight bond.
Human retrieval studies have shown that HA-coated stem observed significantly more ingrowth and attachment of the bone [47].

\section{Conclusion}

In conclusion, $\mathrm{HA}$ is better than porous coating. HA coating could improve the postoperative HHS, reduce the incidence of thigh pain, and reduce the incidence of femoral osteolysis, while there was no statistical difference of femoral stem survivorship from aseptic loosening, polyethylene wear, and radiolucent lines between the two groups.

\section{Abbreviations \\ HA: hydroxyapatite; HHS: Harris hip score; THA: total hip arthroplasty; $\mathrm{RCT}$ : randomized controlled trial; WMD: weighed mean difference; $\mathrm{Cl}$ : confidence intervals; RR: relative risk; BMD: bone mineral density; WOMAC: Western Ontario and McMaster Universities Arthritis Index.}

\section{Competing interests}

The authors declare that they have no competing interests.

\section{Authors' contributions}

CYL and SMM searched the literature. TL, AL, HB, SZL, and YSG reviewed the literature and extracted and analyzed the data. CYL and SMM evaluated the study quality and performed the meta-analysis. CYL and HB drafted the manuscript. LT and SMM revised the manuscript. All authors read and approved the final manuscript.

\section{Acknowledgements}

This work is supported by the National Natural Science Foundation of China (81101377, 81101345, 81171687, 81371954, 81201414, 81201416, 81401785), the Key Project of Zhejiang Provincial Department of Science and Technology (2011C13033), the Zhejiang Provincial Natural Science Foundation of China (Y2100161,Y2090283), the Scientific Research Fund of Zhejiang Provincial Education Department (Y201018936), and the Medical Scientific Research Foundation of Zhejiang Provience of China (2012RCA032).

Received: 7 July 2014 Accepted: 8 January 2015

Published online: 28 January 2015

\section{References}

1. Prieto-Alhambra D, Javaid MK, Judge A, Maskell J, Kiran A, de Vries F, et al. Fracture risk before and after total hip replacement in patients with osteoarthritis: potential benefits of bisphosphonate use. Arthritis Rheum. 2011;63:992-1001.

2. Manley MT, D'Antonio JA, Capello WN, Edidin AA. Osteolysis: a disease of access to fixation interfaces. Clin Orthop. 2002;405:129-37.

3. Kim YH, Park JW, Patel C, Kim DY. Polyethylene wear and osteolysis after cementless total hip arthroplasty with alumina-on-highly cross-linked polyethylene bearings in patients younger than thirty years of age. J Bone Joint Surg Am. 2013;95(12):1088-93.

4. Søballe K, Toksvig-Larsen S, Gelineck J, Fruensgaard S, Hansen ES, Ryd L, et al. Migration of hydroxyapatite coated femoral prostheses. A Roentgen Stereophotogrammetric study. J Bone Joint Surg Br. 1993;75(5):681-7.

5. D'Angelo F, Molina M, Riva G, Zatti G, Cherubino P. Failure of dual radius hydroxyapatite-coated acetabular cups. J Orthop Surg Res. 2008;3:35.

6. Bloebaum RD, Beeks D, Dorr LD, Savory CG, DuPont JA, Hofmann AA. Complications with hydroxyapatite particulate separation in total hip arthroplasty. Clin Orthop. 1994;298:19-26.

7. Geesink RGT, Hoefnagels NHM. Six year results of hydroxyapatite-coated total hip replacement. J Bone Joint Surg Br. 1995;77(4):534-47.

8. Park YS, Lee JY, Yun SH, Jung MW, Oh I. Comparison of hydroxyapatite- and porous-coated stems in total hip replacement. Acta Orthop Scand. 2003;74(3):259-63

9. Parvizi J, Sharkey PF, Hozack WJ, Orzoco F, Bissett GA, Rothman RH. Prospective matched-pair analysis of hydroxyapatite-coated and uncoated 
femoral stems in total hip arthroplasty. A concise follow-up of a previous report. J Bone Joint Surg Am. 2004;86-A(4):783-6.

10. Rothman RH, Hozack WJ, Ranawat A, Moriarty L. Hydroxyapatite-coated femoral stems. A matched-pair analysis of coated and uncoated implants. J Bone Joint Surg Am. 1996;78(3):319-24.

11. Sharp RJ, O'Leary ST, Falworth M, Cole A, Jones J, Marshall RW. Analysis of the results of the C-Fit uncemented total hip arthroplasty in young patients with hydroxyapatite or porous coating of components. J Arthroplasty. 2000;15(5):627-34.

12. Lombardi Jr AV, Berend KR, Mallory TH. Hydroxyapatite-coated titanium porous plasma spray tapered stem: experience at 15 to 18 years. Clin Orthop Relat Res. 2006;453:81.

13. Rasquinha VJ, Ranawat CS, Mauriello Jr AJ. Hydroxyapatite: catalyst or conjuror? J Arthroplasty. 2002;17 Suppl 1:113-7.

14. Santori FS, Ghera S, Moriconi A, Montemurro G. Results of the anatomic cementless prosthesis with different types of hydroxyapatite coating. Orthopedics. 2001;24(12):1147-50.

15. Ciccotti MG, Rothman RH, Hozack WJ, Moriarty L. Clinical and roentgenographic evaluation of hydroxyapatite-augmented and nonaugmented porous total hip arthroplasty. J Arthroplasty. 1994;9(6):631-9.

16. Dorr LD, Wan Z, Song M, Ranawat A. Bilateral total hip arthroplasty comparing hydroxyapatite coating to porous-coated fixation. J Arthroplasty. 1998;13(7):729-36.

17. Gandhi R, Davey JR, Mahomed NN. Hydroxyapatite coated femoral stems in primary total hip arthroplasty: a meta-analysis. J Arthroplasty. 2009;24(1):38-42.

18. Goosen JH, Kums AJ, Kollen BJ, Verheyen CC. Porous-coated femoral components with or without hydroxyapatite in primary uncemented total hip arthroplasty: a systematic review of randomized controlled trials. Arch Orthop Trauma Surg. 2009;129(9):1165-9.

19. Furlan AD, Pennick V, Bombardier C, van Tulder M. Updated method guidelines for systematic reviews in the Cochrane Back Review Group Spine. 2009;34:1929-41.

20. Higgins JPT, Green S. Cochrane Collaboration. Cochrane handbook for systematic reviews of interventions. Chichester, England and Hoboken NJ: Wiley-Blackwell; 2008.

21. Rorabeck C. Tapered hydroxyapatite-coated press-fit stems: any added value? J Arthroplasty. 2006;21 (4 Suppl 1):85-8.

22. Tanzer M, Kantor S, Rosenthall L, Bobyn JD. Femoral remodeling after porous-coated total hip arthroplasty with and without hydroxyapatitetricalcium phosphate coating: a prospective randomized trial. J Arthroplasty. 2001;16(5):552-8.

23. Kim YH, Kim JS, Oh SH, Kim JM. Comparison of porous-coated titanium femoral stems with and without hydroxyapatite coating. J Bone Joint Surg Am. 2003;85-A(9):1682-8.

24. Kim YH, Kim JS, Joo JH, Park JW. Is hydroxyapatite coating necessary to improve survivorship of porous-coated titanium femoral stem? J Arthroplasty. 2012;27(4):559-63.

25. Jaffe WL, Scott DF. Rationale and clinical application of hydroxyapatite coatings in pressfit total hip arthroplasty. Semin Arthroplasty. 1993;4(3):159-66.

26. Geesink RG. Osteoconductive coatings for total joint arthroplasty. Clin Orthop Relat Res. 2002;395:53-65.

27. Egger M, Davey Smith G, Schneider M, Minder C. Bias in meta-analysis detected by a simple, graphical test. BMJ. 1997;315(7109):629-34.

28. Camazzola D, Hammond T, Gandhi R, Davey JR. A randomized trial of hydroxyapatite-coated femoral stems in total hip arthroplasty: a 13-year follow-up. J Arthroplasty. 2009;24(1):33-7.

29. Yoon KS, Kim HJ, Lee JH, Kang SB, Seong NH, Koo KH. A randomized clinical trial of cementless femoral stems with and without hydroxyapatite tricalciumphosphate coating. An 8- to 12-year follow-up study. J Arthroplasty. 2007;22(4):504-8.

30. Yee AJ, Kreder HK, Bookman I, Davey JR. A randomized trial of hydroxyapatite coated prosthesis in total hip arthroplasty. Clin Orthop Relat Res. 1999;366:120-32.

31. Tanzer M, Gollish J, Leighton R, Orrell K, Giacchino A, Welsh P, et al. The effect of adjuvant calcium phosphate coating on a porous-coated femoral stem. Clin Orthop Relat Res. 2004;424:153-60.

32. Incavo SJ, Schnelder R, Elting J. The effect of surface coating of femoral prostheses implanted without cement: a 2- to 4-year follow-up study. Am J Orthop (Belle Mead, NJ). 1998;27(5):355-61.

33. Lee JM, Lee CW. Comparison of hydroxyapatite-coated and nonhydroxyapatite-coated noncemented total hip arthroplasty in same patients. J Arthroplasty. 2007;22(7):1019-23.
34. Hamadouche M, Witvoet J, Porcher R, Meunier A, Sedel L, Nizard R. Hydroxyapatite-coated versus grit-blasted femoral stems, a prospective, randomised study using EBRA-FCA. J Bone Joint Surg Br. 2001;83(7):979-87.

35. Ranawat CS, Rasquinha VJ, Meftah M, Ranawat AS. The hydroxyapatitetapered stem: any added value? Semin Arthroplasty. 2011;22:75-8.

36. McPherson EJ, Dorr LD, Gruen TA, Saberi MT. Hydroxyapatite-coated proximal ingrowth femoral stems. A matched pair control study. Clin Orthop Relat Res. 1995;315:223-30.

37. Sanchez-Sotelo J, Lewallen DG, Harmsen WS, Harrington J, Cabanela ME. Comparison of wear and osteolysis in hip replacement using two different coatings of the femoral stem. Int Orthop. 2004;28(4):206-10.

38. Paulsen $A$, Pedersen $A B$, Johnsen SP, Riis $A$, Lucht $U$, Overgaard S. Effect of hydroxyapatite coating on risk of revision after primary total hip arthroplasty in younger patients: findings from the Danish Hip Arthroplasty Registry. Acta orthop. 2007;78(5):622-8.

39. Sano K, Ito K, Yamamoto K. Changes of bone mineral density after cementless total hip arthroplasty with two different stems. Int Orthop. 2008;32(2):167-72.

40. Brown TE, Larson B, Shen F, Moskal JT. Thigh pain after cementless total hip arthroplasty: evaluation and management. J Am Acad Orthop Surg. 2002:10(6):385-92.

41. Hernández CP, Nájera SO, Mesa RF, Pajares LM, Hernández HM. Hydroxyapatitecoated stems with metaphyseal and diaphyseal press-fit. Eleven-year follow-up results. Acta Orthop Belg. 2002;68(1):24-32.

42. Joshi MG, Advani SG, Miller F, Santare MH. Analysis of a femoral hip prosthesis designed to reduce stress shielding. J Biomech. 2000;33(12):1655-62.

43. Dopico-González C, New AM, Browne M. Probabilistic finite element analysis of the uncemented hip replacement-effect of femur characteristics and implant design geometry. J Biomech. 2010;43(3):512-20.

44. Ando M, Imura S, Omori H, Okumura Y, Bo A, Baba H. Nonlinear threedimensional finite element analysis of newly designed cementless total hip stems. Artif Organs. 1999;23(4):339-46.

45. Herrera A, Mateo J, Lobo-Escolar A, Panisello JJ, Ibarz E, Gracia L. Long-term outcomes of a new model of anatomical hydroxyapatite-coated hip prosthesis. J Arthroplasty. 2013;28(7):1160-6.

46. Cao CF, Zhou JJ, Pang JH, Chen XQ. A five-year clinical and radiographic follow-up of bipolar hip arthroplasty with insertion of a porous-coated anatomic femoral component without cement. Orthop Surg. 2011;3(2):88-94.

47. Coathup MJ, Blunn GW, Flynn N, Williams C, Thomas NP. A comparison of bone remodelling around hydroxyapatite-coated, porous-coated and gritblasted hip replacements retrieved at post-mortem. J Bone Joint Surg Br. 2001;83(1):118-23.

\section{Submit your next manuscript to BioMed Central and take full advantage of:}

- Convenient online submission

- Thorough peer review

- No space constraints or color figure charges

- Immediate publication on acceptance

- Inclusion in PubMed, CAS, Scopus and Google Scholar

- Research which is freely available for redistribution

Submit your manuscript at www.biomedcentral.com/submit
C BioMed Central 